\title{
Experiments in Fixturing Mechanics
}

\author{
Joel W. Burdick and Yongqiang Liang \\ $\{j w b$,liang $\} @$ robotics.caltech.edu \\ Mechanical Engineering \\ California Institute of Technology, Pasadena, CA
}

\author{
Elon Rimon \\ elon@robbby.technion.ac.il \\ Mechanical Engineering \\ Technion, Haifa, Israel
}

\begin{abstract}
This paper describes an experimental fixturing system wherein fixel reaction forces, workpiece loading, and workpiece displacements are measured during simulated fixturing operations. The system's configuration, its measurement principles, and tests to characterize its performance are summarized. This system is used to experimentally determine the relationship between workpiece displacement and variations in fixel preload force or workpiece loading. We compare the results against standard theories, and conclude that commonly used linear spring models do not accurately predict workpiece displacements, while a non-linear compliance model provides better predictive behavior.
\end{abstract}

\section{INTRODUCTION}

Fixturing is an essential part of flexible manufacturing systems. The quality of an automatically generated fixture plan is crucially dependent upon the accuracy of the underlying physical models upon which the plan is based. Inaccurate models can lead to poor prediction of fixture performance, as well as costly and time consuming iterations on fixture system design. This is particularly true for complex workpieces and applications involving high forces or requiring tight tolerances.

As reviewed below, a number of theories have been proposed for predicting the behavior of fixtured workpieces under loading. This paper presents an experimental system (Fig. 1) that can test many of these theories. Our experimental system measures fixel reaction forces, workpiece loads, and workpiece displacements during simulated fixturing operations. The system design, its measurement principles, and experiments to characterize its performance are described. Using this system, we experimentally explore the relationship between workpiece displacement and variations in fixel preloading or variations in workpiece loading. We compare the results against the predictions of four different models, thereby testing the predictive quality of these models. We conclude that the widely used linear spring compliance model does not accurately predict trends in workpiece displacement, while a nonlinear stiffness model [17], [18], [28] provides better predictions.

Relation to Prior Work. We use innovative fixture hardware to verify the physical theories that underlie automated fixture planning. Prior fixture hardware innovations have mainly involved conformable [3], [23] and modular fixtures [7], [20]. However, prior fixturing prototypes have not been sufficiently instrumented to test all of the key

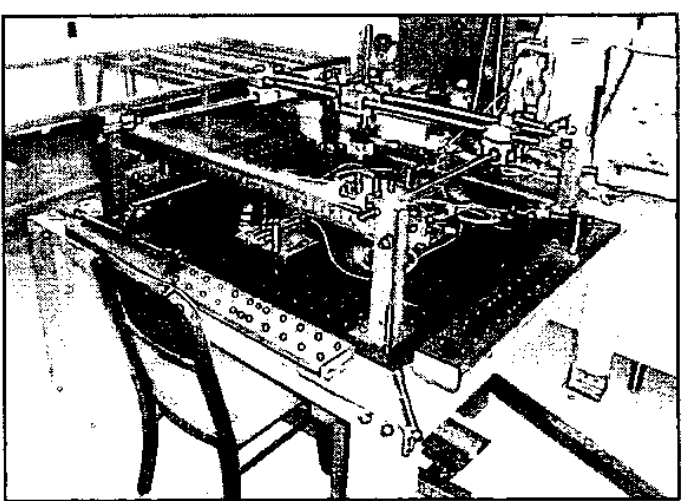

Fig. 1. Photograph of overall system.

mechanical relationships in the fixturing process. Previous investigators have instrumented a single contact [2], [32], or have measured either fixture reaction forces or workpiece displacements [9]. Our multi-contact system measures the fixel reaction forces, workpiece load, and the displacement of points in the workpiece due to loading.

Our goal in measuring these variables is to determine which theories better predict the behavior of fixtures under realistic loading situations. We are particularly concerned with applications involving high forces and/or tight tolerances-cases where good predictive capability is required. With the insight from these experiments, the underlying physical basis for automated fixture planning systems can potentially be improved.

Automated fixture planning systems can be knowledgeor analysis-based. Knowledge-based planning systems rely on AI techniques to select fixture elements, fixel positions, and clamping forces [5], [6], [21]. Since these methods are not based upon physical principles, the resulting designs are not guaranteed to meet functional requirements. Analysisbased fixture planning is built upon physical models of fixture mechanics. With proper analytical models, one can test if the resulting designs can meet functional requirements.

In the most common analysis-based paradigm, the workpiece and fixels are treated as rigid bodies. Fixture plans often ensure that the fixture achieves force closure. However, force-closure fixtures can be statically indeterminate: given an applied wrench, the equilibrium equations do not uniquely determine fixel reaction forces [30]. Additional, 
possibly unrealistic, assumptions are usually needed in rigid body fixture force analysis [9], [13], [15].

Rigid body fixture planning can also be based on formclosure. Rimon and Burdick have identified $1^{\text {st }}$-order and $2^{\text {nd }}$-order form closure grasps [27]. Second-order form closure employs curvature effects to realize fixture immobility. Several methods have been proposed for synthesizing firstorder form-closure fixtures (e.g. [31], [33]). The planning of second-order form-closure was considered in [25].

Fixtures used in machining operations can experience large cutting forces (e.g., $\sim 5000 \mathrm{lbf}[8]$ ). While forceclosure that relies on friction is often used in robotic grasping, it is less often used for workpiece fixturing except in light-duty applications, or for vises and chucks [1]. Moreover, friction can be hard to estimate without knowing the amount of cutting fluid and grease [4]. We focuse on fixtures that do not rely upon friction, as they are most relevant to high force and tight tolerance applications.

While rigid-body models are acceptable for some fixturing applications, they are inadequate in many high force applications, where the workpieces usually experience nonnegligible deformations [10], [9] that must be analyzed and controlled to ensure machining accuracy. Similarly, workpiece and fixture strength must be analyzed to avoid structural damage. Deformations cannot be determined from rigid-body models, and their static indeterminacy complicates the computation of reaction forces and evaluation of material strength.

Closed form lumped-parameter compliance models are efficient and well-suited for automated fixture planning. The linear spring model is simplest and most widely used model this type. It represents a compliant contact as a linear spring acting along a fixed direction on a rigid object [22], [24]. This model has many shortcomings. Foremost, the linear-spring model is not supported by elasticity theory or experimental data. Thus, it cannot provide the accurate predictions that are essential for precision fixturing. Second, systematic procedures have not been proposed to position the linear-spring elements that model contact compliance, or to compute the spring stiffness coefficients. For fixture planning algorithms that should accurately compute fixture arrangements, reaction forces, and workpiece deffections from CAD models, these shortcomings are significant.

Finite element models [16], [29] and analogous ideas [11] have been used to analyze workpiece deformations and fixture stresses. While accurate, these numerical approaches have drawbacks. The relationship between fixture reaction forces and object displacement can only be obtained numerically. Hence, the stiffness matrix, which is often used as the basis for fixture optimization procedures, can only be found through numerical means. Thus, while such numerical methods are useful for verifying fixture designs, they are not well-suited for automated fixture planning.

In summary, the most practically useful physical models for automated fixture planning are lumped parameter compliances models. Our experimental system was devised to test these types of compliance theories.

\section{EXPERIMENTAL APPARATUS}

For simplicity, our system is restricted to planar arrangements. We wish to measure the variables needed to evaluate relevant compliance theories (Section IV). To focus on the effects of workpiece and fixel surface compliance, we overdesigned the supporting structures to minimize their contribution to overall system stiffness, and used appropriate measurement techniques (see below). Our system can work with metal and plastic objects up to 15 inches wide and 2 inches thick. As reviewed below, the sizes of the applied loads and fixel reaction forces are consistent with nominal practice [9].

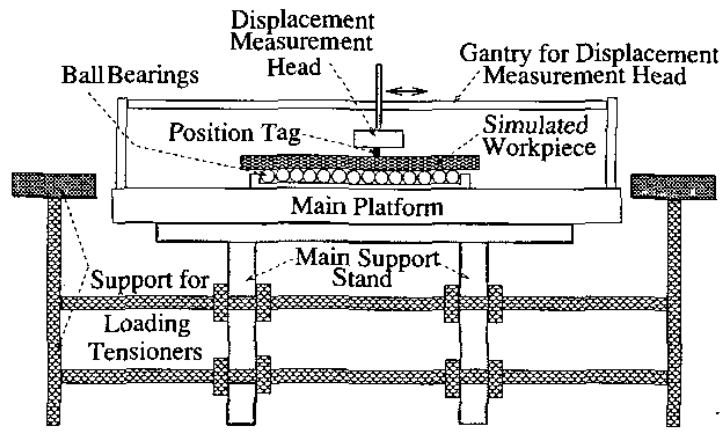

Fig. 2. Schematic cross-sectional view of experiment, without subsystems depicted in Fig.s 3 and 5. The drawing is not to scale.

Due to its many components, schematic views of the system are divided across Fig.s 2, 3, and 5. The main platform consists of a 42 " square by 2 " thick ground solid steel plate (weighing $\sim 500 \mathrm{~kg})$. This plate is drilled $(0.5 "$ depth) and tapped on $3^{\prime \prime}$ centers with $\frac{1}{2} " \times 20$ holes for bolting down fixels in various configurations. The platform rests on a welded steel frame $(\sim 200 \mathrm{~kg}$ mass $)$, which sits on a floor that lies upon solid bedrock. See Fig. 2 for a schematic of the platform and support structures.

Fixels. Each fixel consists of a solid removable fingertip attached to a power screw, which in turn is mounted on a structure whose position and orientation can be adapted to different workpiece arrangements (see Fig.s 3 and 4) . Finger tips made with different materials and different radii of curvature enable the effects of fixel geometry and material choice to be tested.

In order to focus on compliance effects due to deformations in the vicinity of the contacts, very stiff quartz sensors (Kistler model 9212, coupled to Kistler charge amplifiers) are used to measure the fixel normal reaction forces ${ }^{1}$. These sensors can measure forces up to $5,000 \mathrm{lbf}$ with a linearity of $0.5 \%$. Due to their high stiffness, these sensors

\footnotetext{
${ }^{1}$ The system currently measures normal reaction forces. Plans are
} underway to enable direct measurement of tangential forces. 


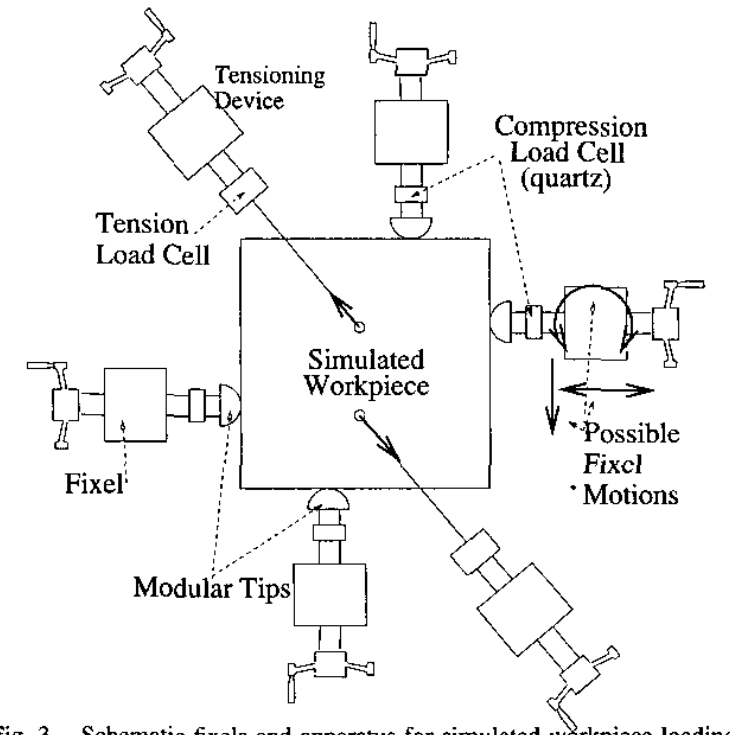

Fig. 3. Schematic fixels and apparatus for simulated workpiece loading.

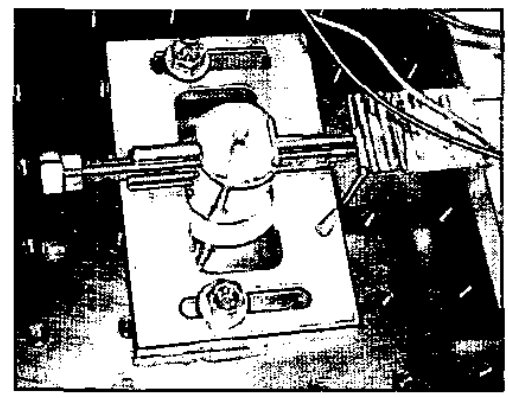

Fig. 4. Photograph of fixel element

only modify overall system compliance by $3-5 \%$ in the worst of case steel fixel tips. In contrast, the flexible substructures on which conventional strain gauges are mounted would dominate overall fixture system compliance. Our design avoids this problem.

Workpiece Loading. Static workpiece loading can be simulated as follows. Two power screws create tension in steel rods that are attached to the workpiece (see Fig. 3) using gimballed eye-bolts placed over two support pegs. Each simulated workpiece must be modified to allow loading in this manner. Since the tensioners can be placed arbitrarily around the workpiece, their combined actions can generate any net planar wrench on the workpiece. To minimize the influence of workpiece loading on the displacement measurement process, the tensioning devices are mounted on a separate outer structure (see Fig. 2). In this way, simulated workpiece loads do not warp the platform under the workpiece, thereby causing displacement measurement errors. The applied wrench can be calculated from the rod tensions, which are measured by load cells (that can measure forces up to 1,000 lbf with $0.5 \%$ linearity).

Workpiece Displacement Measurement. As discussed

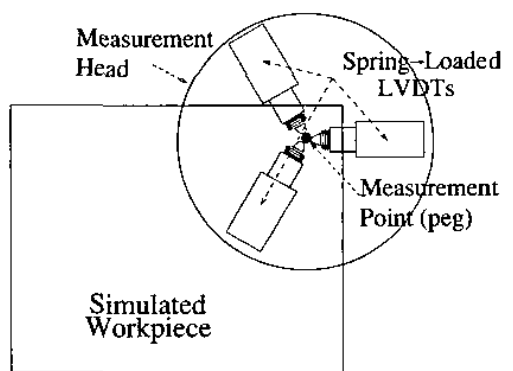

Fig. 5. Schematic of workpiece displacement measurement system.

in Sections IV and $\mathrm{V}$, lumped parameter compliance models can predict the displacement of workpiece points in response to loading. Our system measures these displacements using Linear Variable Differential Transformer (LVDT) sensors. This technique is a low-cost alternative to laser-based displacement measurement that has acceptable accuracy. LVDT sensors measure displacement along one axis. We employ three LVDTs in a redundant triangular arrangement (Fig. 5) to more accurately measure displacements. To measure the displacement of a workpiece point, the workpiece must be modified with a small peg, or "position tag" at the point of interest. At the beginning of the experiment, a gantry supported "measurement head" containing the (lightly spring-loaded) LVDTs is lowered over the position tag. Micrometers are used to adjust and zero the LVDTs' positions. The LVDTs measure displacement from this initial position in response to variations in workpiece or fixel forces that are subsequently applied. Two measurement ranges are available: up to 200 microns displacement with a 0.1 micron resolution, or up to 1000 microns displacement with 0.5 micron resolution.

Support of the Simulated Workpiece. To accurately test theoretical predictions, the workpiece should only experience fixel loading and workpiece loading forces. To eliminate any frictional reaction forces that might occur between the workpiece and the system platform, the workpiece rests on a bed of ball-bearings (see Fig. 2).

\section{SYSTEM CHARACTERIZATION}

We summarize here the outcomes of experiments that were aimed at determining the inherent noise, variability, accuracy, and repeatability of this system.

Displacement measurement errors. Errors in measuring workpiece displacement can arise from several sources: noise in the LVDTs, background vibrations of the teststand, creep in the support structures, and structural warping during workpiece loading. We performed a series of experiments to characterize these effects. First, unloaded LVDT signals were sampled to determine the baseline noise amplitude (equivalent to 0.1 micron of workpiece displacement). In others tests, the LVDT signals were sampled for extended periods while the measurement head was placed on a position tag of an unloaded workpiece. This test 
revealed mechanical creep in the measurement head gantry and support system for the first $\sim 20$ minutes after placing the measurement head. This creep; was equivalent in the worst cases to $\sim 2$ microns of displacement. Additionally, steady state background vibrations had a peak amplitude of 0.7 microns. Other tests showed that due to the high stiffness of the main platform, and the separation of the loading tensioners' support (Fig. 2), variations in tensioner loads cause no measurable amounts of error.

Displacement measurement variability. To test the repeatability of our experimental procedure, we loaded a workpiece (see Section $V$ for its description) into the system, zeroed the LVDTs, applied a fixed wrench, and measured the resulting displacement. We repeated this process, dismantling the fixture before each trial. These experiments show that over the loading range of interest, our procedure is repeatable to $\sim 4$ microns of displacement.

Load Cells. We found the factory calibrations of the fixel and tensioner load cells to be accurate within our measurement capabilities.

In summary, for the conditions of interest, noise, creep, and loading contribute up to $\sim 6$ microns of error in displacement measurement accuracy, and the setup and loading procedure can cause up to $\sim 5$ microns in nonrepeatability. In a typical experiment, the position tag displaces by a few hundred microns.

\section{Compliance Modelling Review}

To motivate the ensuing experiments, we briefly review lumped parameter, or quasi-rigid body, compliance models. For more details, see [17], [18], [19]. A fixture consists of an object $\mathcal{B}$ contacted by $k$ fixels $\mathcal{A}_{1}, \ldots, \mathcal{A}_{k}$ (e.g., Fig. 7). Because we focus on fixtures that do not rely upon friction for their success, we initially assume that the contacts are frictionless. The bodies' surfaces are assumed smooth at the contacts. We assume that the bodies are quasi-rigid, and that the fixturing elements are stationary. In the quasirigid assumption, deformations due to compliance effects are assumed to be localized in the vicinity of the contacts, so that $\mathcal{B}$ 's overall motion relative to $\mathcal{A}_{i}$ can be described using rigid-body kinematics. For objects without slender substructures, the quasi-rigid approximation is sound.

$\mathcal{B}$ 's configuration space (c-space) is parametrized by the coordinates $q=(d, \theta)$, where $d \in \mathbf{R}^{2}$ is $\mathcal{B}$ 's position and $\theta \in \mathbb{R}$ denotes $\mathcal{B}$ 's orientation. Velocities take the form $\dot{q}=$ $(v, \omega)$, where $v$ and $\omega$ are the linear and angular velocities of $\mathcal{B}$. Wrenches take the form $w=(f, \tau)$, with $f$ and $\tau$ the force and torque acting on $\mathcal{B}$.

The Overlap Representation. In quasi-rigid body modelling, the details of the compliant surface deformations are ignored, and the contact forces are modelled as a function of $\mathcal{B}$ 's relative movement. Rimon and Burdick [28] have shown how this can be achieved using overlap functions. Let $\mathcal{B}(q)$ be the subset of $\mathbf{R}^{2}$ occupied by the undeformed
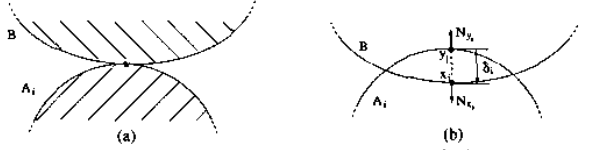

Fig. 6. (a) An initial point contact. (b) After a relative approach. of $\delta_{i}$.

shape of $\mathcal{B}$ at configuration $q$. Rather than solve for the complex surface deformations that arise during compliant interaction, imagine that the rigid shapes of $\mathcal{B}$ and $\mathcal{A}_{i}$ freely interpenetrate during the movement that accompanies their true compression. The overlap between $\mathcal{B}(q)$ and $\mathcal{A}_{i}$, denoted $\delta_{i}(q)$, is the minimum amount of translation that would separate $\mathcal{B}$ from $\mathcal{A}_{i}$. When $\mathcal{B}(q)$ overlaps $\mathcal{A}_{i}$, the overlap segment has endpoints $x_{i}$ and $y_{i}$ on the boundaries of $\mathcal{B}$ and $\mathcal{A}_{i}$ such that $\delta_{i}=\left\|x_{i}-y_{i}\right\|$ (Fig. 6). The surface normals at $x_{i}$ and $y_{i}$, denoted $N_{x_{i}}$ and $N_{y_{i}}$, are collinear.

The overlap $\delta_{i}$ is the relative approach of the bodies used in the contact mechanics literature [14]. Also in agreement with this literature, the compliant interaction forces are lumped into a single force collinear with the overlap segment $\overline{x_{i} y_{i}}$ and having magnitude $F_{i}=f_{i}\left(\delta_{i}(q)\right)$. The function $f_{i}$ determines the compliance relationship between $\delta_{i}$ and $F_{i}$. Different choices of $f_{i}$ yield the linear spring model $\left(f_{i}=k_{i} \delta_{i}\right)$, or the Hertz contact model.

Hertz Contact Model. When two bodies are brought into contact they touch initially at a point (3D case) or a line (2D case for cylindrical bodies). Under the action of the load, they deform and touch over a finite contact area surrounding the point or line of first contact. Using the assumption that the contact area is an ellipse with principal semi-axes $a$ and $b$, Hertz derived a relationship between the loading force $F_{i}$ and the relative approach $\delta_{i}$ for $3 \mathrm{D}$ contacts. The Hertzian compliance relationship (for 3D frictionless bodies) is:

$$
F_{i}=\frac{4}{3} \beta_{1}(e) E^{*}\left(r_{r e l_{1}} r_{r e l_{2}}\right)^{1 / 4} \delta_{i}^{\frac{3}{2}}
$$

where $E^{*}=\left(\frac{1-\nu_{A}^{2}}{E_{A}}+\frac{1-\nu_{B}^{2}}{E_{B}}\right)^{-1}$, with $E_{A}, E_{B}$ the Young's moduli and $\nu_{A}, \nu_{B}$ the Poisson's ratios of $\mathcal{A}_{i}$ and $\mathcal{B}$. The principal radii of relative curvature $r_{r e l_{1}}$ and $r_{r e l_{2}}$ are the eigenvalues of $L_{r e l}^{-1}$. The relative curvature matrix $L_{\text {rel }}$ is $L_{\text {rel }}=L_{\mathcal{A}_{i}}+L_{\mathcal{B}_{i}}$ where $L_{\mathcal{A}_{i}}$ and $L_{\mathcal{B}_{i}}$ denote the curvatures of $\mathcal{A}_{i}$ and $\mathcal{B}$ at the $i^{t h}$ contact. The term $\beta_{1}(e)$ is a function of elliptic integrals of the parameter $e=\left(1-b^{2} / a^{2}\right)^{1 / 2}$, where $a$ and $b$ in turn depend upon the bodies' curvatures. Since (1) takes the form $F_{i}=k_{i} \delta_{i}^{3 / 2}$, the Hertz contact model corresponds to a particular choice of $f_{i}$ in the overlap model.

The line contact problem, which is relevant for the planar bodies in our experiments, models bodies by cylinders whose cross section is the planar shape. Unfortunately, in this case elasticity theory yields a force-displacement relationship that depends upon the choice of datum points. Choosing datum points for each body at distances $l_{\mathcal{B}}, l_{\mathcal{A}_{i}}$ from the contact point (along the contact normals), the 
overlap is related to the interaction force $F$ by:

$$
\delta_{i}=F_{i}\left[\alpha_{B} \ln \left(\frac{\eta_{B} l_{\mathcal{B}}}{F_{i}}\right)+\alpha_{A} \ln \left(\frac{\eta_{A} l_{\mathcal{A}_{i}}}{F_{i}}\right)\right]
$$

where $\alpha_{A}=\left(1-\nu_{A}^{2}\right) /\left(\pi E_{A}\right), \alpha_{B}=\left(1-\nu_{B}^{2}\right) /\left(\pi E_{B}\right)$, $\eta_{A}=\pi E^{*} /\left(4 r_{r e l} \exp \left(\nu_{A} /\left(1-\nu_{A}\right)\right)\right)$, and $\eta_{B}=$ $\pi E^{*} /\left(4 r_{r e l} \exp \left(\nu_{A} /\left(1-\nu_{A}\right)\right)\right)$. The compliance function is the inverse of this relation.

Computation of the Stiffness Matrix. The stiffness matrix is a primary tool for characterizing the compliant behavior and stability of a fixture, and is frequently used in fixture planning [12], [17]. The stiffness matrix for any overlap-based model can be derived as follows. The potential energy of the elastic system consisting of $\mathcal{B}$ and fixels $\mathcal{A}_{1}, \ldots, \mathcal{A}_{k}$ can be expressed as

$$
\Pi(q)=\sum_{i=1}^{k} \int_{0}^{\delta_{i}(q)} f_{i}(\delta) d \delta
$$

For a sufficiently small displacement of $\mathcal{B}$ approximated by a rigid-body velocity $\dot{q}$ at $q_{0}$, the net fixture reaction wrench is given by $w=K \dot{q}$, where $K=D^{2} \Pi\left(q_{0}\right)$ is the stiffness matrix, whose formula is (with $f_{i}^{\prime}=\frac{d f_{i}}{d \delta_{i}}$ ):

$$
\begin{aligned}
K & =\sum_{i=1}^{k} f_{i}^{\prime}\left(\delta_{i}\left(q_{0}\right)\right) \nabla \delta_{i}\left(q_{0}\right) \nabla \delta_{i}\left(q_{0}\right)^{T} \\
& +\sum_{i=1}^{k} f_{i}\left(\delta_{i}\left(q_{0}\right)\right) D^{2} \delta_{i}\left(q_{0}\right) .
\end{aligned}
$$

The coefficient $f_{i}\left(\delta_{i}\left(q_{0}\right)\right)$ describes the pre-load stiffening effects. Formulas for $\delta_{i}\left(q_{0}\right), \nabla \delta_{i}\left(q_{0}\right)$, and $D^{2} \delta_{i}\left(q_{0}\right)$ in the 3-dimensional case can be found in [17], [18], [19]. For planar fixtures (that are relevant to our experiment), $\nabla \delta_{i}\left(q_{0}\right)=-\left[N_{i}^{T} \rho_{i}\right]$, where $\rho_{i}=x_{i} \times N_{i}$. The formula for $D^{2} \delta_{i}\left(q_{0}\right)$ in the 2-D case is:

$$
\frac{1}{r_{\overline{A_{i}}}+r_{\mathcal{B}_{i}}}\left[\begin{array}{cc}
\left(N_{i} N_{i}^{T}-I\right) & \left(r_{\mathcal{B}_{i}}-\rho_{i}\right) J N_{i} \\
\left(r_{\mathcal{B}_{i}}-\rho_{i}\right)\left(J N_{i}\right)^{T} & \left(r_{\bar{A}_{i}}+\rho_{i}\right)\left(r_{\mathcal{B}_{i}}-\rho_{i}\right)
\end{array}\right]
$$

where $r_{\mathcal{B}_{i}}$ is the radius of $\mathcal{B}$ 's boundary at the $i^{\text {th }}$ contact, $r_{\overline{A_{i}}}=r_{A_{i}}-\delta_{i}\left(q_{0}\right)$, with $r_{A_{i}}$ being the radius of the $i^{\text {th }}$ fixel at the contact, $N_{i}$ the contact normal, and $J=\left(\begin{array}{cc}0 & 1 \\ -1 & 0\end{array}\right)$.

\section{EXPERIMENTAL GEOMETRY}

Fig. 7 shows the geometry of a fixture for which experimental results are given. Four cylindrical fixels, with identical radii of curvature $r_{A}=12$ inches, contact the four edges of a rectangular workpiece. The fixel tips are 2 inches thick, and the workpiece is 1 inch thick. Hence, the objects touch in a cylindrical line contact. For convenience, hereafter denote $\delta\left(q_{0}\right)=\delta_{0}$. The fixels are made of the same material, so that $f_{i}\left(\delta_{i}\left(q_{0}\right)\right)=f\left(\delta_{0}\right)$ for all $i$. All fixels are uniformly preloaded to $F_{p r e}=f\left(\delta_{0}\right)$. A position tag is located at coordinates $\left(s_{x}, s_{y}\right)=\left(2.15^{\prime \prime}, 4.15^{\prime \prime}\right)$.

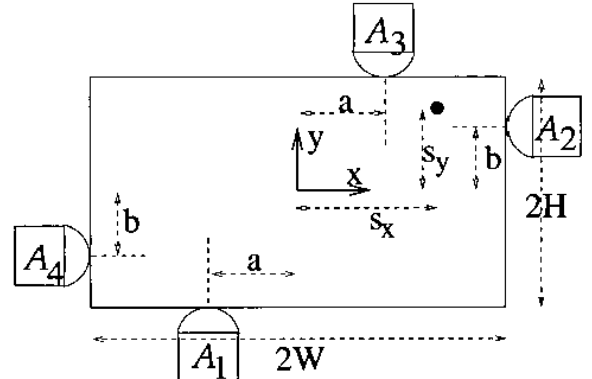

Fig. 7. Geometry of experimental workpiece.

The stiffness matrix for this workpiece is:

$$
K=2\left[\begin{array}{ccc}
f^{\prime}\left(\delta_{i}\left(q_{0}\right)\right) & 0 & 0 \\
0 & f^{\prime}\left(\delta_{i}\left(q_{0}\right)\right) & 0 \\
0 & 0 & Q
\end{array}\right]
$$

where $Q=f^{\prime}\left(\delta_{i}\left(q_{0}\right)\right)\left(a^{2}+b^{2}\right)+F_{p r e}\left(2 r_{A}+a-b\right)$. The displacement, $\left[\begin{array}{cc}\Delta x & \Delta y\end{array}\right]^{T}$, of the position tag under the influence of an applied wrench $W=\left[\begin{array}{lll}F_{x} & F_{y} & \tau\end{array}\right]^{T}$ is:

$$
\left[\begin{array}{c}
\Delta x \\
\Delta y
\end{array}\right]=\left[\begin{array}{ccc}
1 & 0 & -s_{y} \\
0 & 1 & s_{x}
\end{array}\right] K^{-1} W=\frac{1}{2}\left[\frac{\frac{F_{x}}{f^{\prime}\left(\delta_{i}\left(q_{0}\right)\right)}-\frac{\tau s_{y}}{Q}}{f^{\prime}\left(\delta_{i}\left(q_{0}\right)\right)}+\frac{\tau s_{x}}{Q}\right]_{(5)}
$$

We want to evaluate different compliance relationships. First consider the case where $f(\delta)$ follows a power-law relation: $f(\delta)=\mathrm{k} \delta^{p}$ for some $p$. Hence, $f^{\prime}=k p \delta^{p-1}$. Solving for $\delta_{0}$ in terms of the preload yields $\delta_{0}=$ $k^{-1 / p} F_{\text {pre }}^{1 / p}$. Using these relations, one obtains

$$
f^{\prime}\left(\delta_{0}\right)=p k^{1 / p} F_{p r e}^{\left(\frac{p-1}{p}\right)} .
$$

When $p=1$, Eq. (6) represents the Linear Spring model, while Eq. (6) represents the Hertz contact model when $p=$ $\frac{3}{2}$. For other values of $p$, we say that Eq. (6) is a generalized Power Law relationship. For the line contact condition of Eq. (2), the relationship is:

$$
f^{\prime}\left(\delta_{0}\right)=\left(\frac{\partial \delta}{\partial f}\right)_{f\left(\delta_{0}\right)=F_{p r e}}^{-1}=\left[C_{1}-C_{2} \ln \left(F_{p r e}\right)\right]^{-1}
$$

where $C_{1}=\alpha_{B} \ln \left(\eta_{B} l_{\mathcal{B}}\right)+\alpha_{A} \ln \left(\eta_{A} l_{\mathcal{A}}\right)-\left(\alpha_{A}+\alpha_{B}\right)$ and $C_{2}=\alpha_{A}+\alpha_{B}$.

\section{EXPERIMENTAL RESULTS}

We can use this fixture arrangement and Eq.s (5), (6), and (7) to evaluate our experimental procedure and some proposed compliance relationships. Note that the fixture geometry and the nature of the experiments described below were selected to minimize the effects of contact friction. In this way, we can evaluate frictionless contact compliance theories in the real world.

Experiment 1. When a pure force $F_{y}$ is applied in the $y$-direction, Eq. (5) predicts that the total workpiece displacement, $\Delta=\sqrt{(\Delta x)^{2}+(\Delta y)^{2}}$, will vary as:

$$
\Delta=\frac{\left\|F_{y}\right\|}{2 f^{\prime}\left(\delta_{0}\right)} \text {. }
$$


This formula predicts that workpiece displacement will be a linear function of the applied force for all models. However, the effect of preload variation on displacement is a function of the compliance model choice. Based on Eq.s (5), (6), and (7) the Linear Spring, Hertz, Power law, and Cylindrical contact models predict the following relationships between displacement and preload.

$$
\begin{aligned}
& \Delta_{\text {Linear }} \propto\left\|F_{y}\right\|, \quad \Delta_{\text {Hertz }} \propto\left\|F_{y}\right\| F_{p r e}^{-\frac{1}{3}}, \\
& \Delta_{\text {Power }} \propto\left\|F_{y}\right\| F_{p r e e}^{\frac{1-p}{p}} \\
& \Delta_{C y l i n} \propto\left\|F_{y}\right\|\left[C_{1}-C_{2} \ln \left(F_{\text {pre }}\right)\right]
\end{aligned}
$$

Note that the Linear Spring model predicts no variation in workpiece displacement due to preload variations.

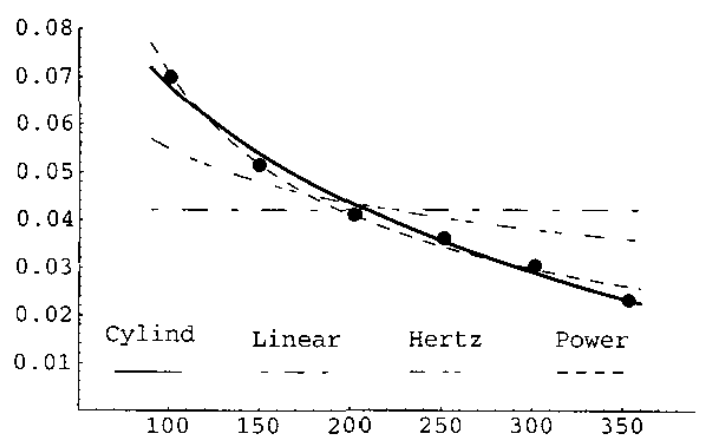

Fig. 8. Workpiece displacement (mm) vs. preload in $\mathrm{kg}\left(\left\|F_{y}\right\|\right.$ fixed at $216 \mathrm{~kg}$ ). Dots are experimental data. Curves are fits of Cylindrical, Linear Spring, 3D Hertz, and Power law (with $p=4.67$ ) contact models.

Fig. 8 shows experimental data, with the different models fit to the data. Since our workpiece and fixels contact along a line, it is not surprising that the Cylindrical line contact model does an excellent job of explaining the experimental data. The power-law model also does a very good job, although the good fit is likely a coincidence. The calculated power of $p=4.67$ has no physical explanation. However, it suggests that when computing the stiffness matrix according to Eq. (3), this power-law is a convenient closedform formula substitute for the inverse of relationship (2), which otherwise has no closed-form formula. Clearly, the Linear Spring model is completely inadequate. The 3D point-contact Hertz model is also inadequate. However, we would expect it to have excellent predictive capabilities when the fixel and object experience true point contact.

Experiment 2. Here we apply a pure torque to the workpiece. Substituting $F_{x}=F_{y}=0$ into Eq. (5), the total displacement of the position tag is

$$
\Delta=\frac{\tau \sqrt{s_{x}^{2}+s_{y}^{2}}}{2 Q}=\frac{\tau S}{2 Q}
$$

where $S=\sqrt{s_{x}^{2}+s_{y}^{2}}$. This formula predicts that for fixed preloading, workpiece displacement should be linear in the applied torque magnitude. When $a=b=0$,
$Q=4 r_{A} F_{p r e}$. I.e, the variation with respect to preload is strictly a geometric effect that is independent of model choice. Fig. 9 shows the displacement vs. preload response when $a=b=2$ ". In this case, the formula for $\Delta$ is weakly dependent upon model selection. The model predictions are superimposed on the data. While the cylindrical contact model continues to provide the best prediction, the other models do a reasonable job since this loading condition is largely model independent. Fig. 10 shows a torque vs. displacement curve (fixel preload is $640 \mathrm{~kg}$, and fixels placed so that $a=b=0$ ) for pure torque loading. The relationship is quite linear in this representative example. While these results do not shed much new light on the proper choice of compliance models, they do confirm the excellent operation of our experimental system.

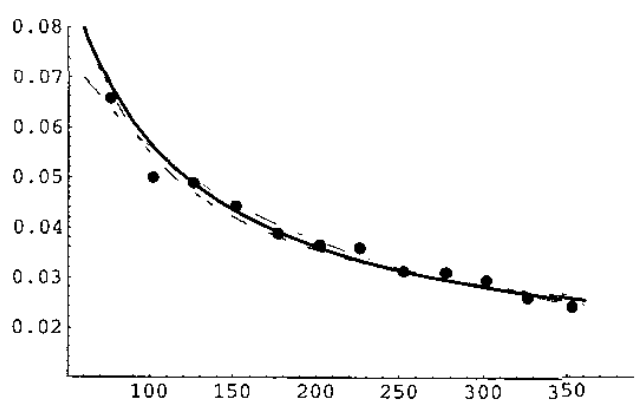

Fig. 9. Workpiece displacement ( $\mathrm{mm}$ ) vs. preload in $\mathrm{kg}$ (torque held fixed). Dots are experimental data. Curves are fits of Cylindrical, Linear Spring, and 3D Hertz contact models (same data scheme as Fig. 8).

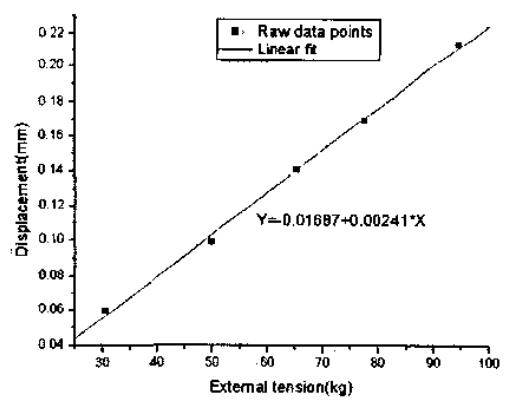

Fig. 10. Workpiece displacement vs. torque.

\section{DISCUSSION}

We presented a novel multi-contact fixturing system that measures the variables which are needed to evaluate the predictive behavior of quasi-rigid body compliance models. Careful design of the system's mechanical structure, sound measurement principles, and extensive system characterization have led to a system that can quantitatively study these issues. We studied frictionless contact quasi-rigid body compliance models because they are relevant to high force fixturing applications. Our experiments show that the 
overlap modelling scheme of [26], [28], [18], coupled with the correct assumptions on contact geometry (point vs. line) can provide excellent predictive capabilities. Conversely, the popular linear spring model gave poor predictions. Clearly, friction is an important issue in contact mechanics. We are currently developing new friction-based non-linear compliance models, and upgrading our device to measure frictional reaction forces.

Acknowledgements: This work was supported by NSF Grant \# IIS-9901056. Many thanks are due to N. Senchy, D. Jackson, J. Burgess, D. Choi, N. Schara, A. Cashion, P. Novak, and A. Duello for designing, constructing, and assembling the experimental system.

\section{REFERENCES}

[1] Y.-C. Chou, V. Chandru, and M. M. Barash. A mathematical approach to automatic configuration of machining fixtures: Analysis and synthesis. ASME J. of Engineering for Industry, 111:299-306, 1989.

[2] M. Cutkosky, P. Akella, R. Howe, and 1. Kao. Grasping as a contact sport. In Proc. Int. Conf. on Robotics and Automation, pages 199-206, 1988.

[3] M. R. Cutkosky, E. Kurokawa, and P. K. Wright. Programmable conformable clamps. In Proc. AUTOFACT 4, pages 1151-1158, 1982.

[4] J. P. Donoghue, W. S. Howard, and V. Kumar. Stable workpiece fixturing. In Proc. ASME Design Technical Conferences, Minneapolis, MN, Sept. 1994.

[5] P. Englert and P. Wright. Applications of artificial intelligence and the design of fixtures for automated manufacturing. In Proc. Int. Conf. on Robotics and Automation, pages 345-351, San Francisco, CA, 1986.

[6] P. M. Ferreria, B. Kochar, C. R. Liu, and V. Chandra. Aifix: an expert systems approach to fixture design. In $A S M E$ Winter Annual Meeting, Computer-Aided/Intelligent Process Planning, pages 73-82, 1985.

[7] A. Friedman. The modular fixturing system, a profitable investment. In Proc. Int. Conf. on Advances in Manufacturing, pages 165-173, 1984.

[8] F. B. Hazen and P. K. Wright. Workholding automation: Innovations in analysis, design, and planning. Manufacturing Review, 3(4):224-237, 1990.

[9] M. Hockenberger and E. De Meter. Preliminary experimentation and modeling of workpiece displacement during machining. NAMRI/SME, 21:351-357, 1993.

[10] M. J. Hockenberger and E. C. De Meter. Effect of machining fixture design parameters on workpiece displacement. Manufacturing Review, 8(1):22-32, 1993.

[11] W. S. Howard and V. Kumar. A minimum principle for the dynamics analysis of systems with frictional contacts. In Proc. Int. Conf. on Robotics and Automation, pages 437442, Atlanta, GA, May 1993.

[12] W. S. Howard and V. Kumar. Stability of planar grasps. In Proc. Int. Conf. on Robotics and Automation, pages 2822 2827, San Diego, CA, May 1994.

[13] S.-L. Jeng, L.-G. Chen, and W.-H. Chieng. Analysis of minimum clamping force. Int. J. of Machine Tools and Manufacture, 35:1213-1224, 1995.

[14] K. L. Johnson. Contact Mechanics. Cambridge University Press, 1985.
[15] L. S.-B. King and F. F. Ling. Force analysis based analytical framework for automatic fixture configuration. In Proc. ASME Int. Mechanical Eng. Congress and Exposition, pages 789-800, New York, NY, 1995.

[16] J. D. Lee and L. S. Haynes. Finite-element analysis of flexible fixturing system. ASME $J$. of Engineering for Industry, 109:134-139, 1987.

[17] Q. Lin, J. W. Burdick, and E. Rimon. Computation and analysis of compliance in grasping and fixturing. In Proc. Int. Conf. on Robotics and Automation, pages 93-99, Albuquerque, NM, April 1997.

[18] Q. Lin, J.W. Burdick, and E. Rimon. Computation and analysis of natural compliance in fixturing and grasping arrangements. IEEE Trans. on Robotics and Automation (to appear), 2003.

[19] Qiao Lin. Mechanics and Planning of Workpiece Fixturing and Robotic Grasping. PhD thesis, California Institute of Technology, Dept. of Mechanical Engineering, June 1998.

[20] B. Madewell. The flexibility of modular fixturing. Cutting Tool Engineering, 48(7), 1996.

[21] A. Y. C. Nee, N. Bhattacharyya, and A. N. Poo. Applying AI in jig and fixture design. Robotics and Computer Integrated Manufacturing, 3(2):195-200, 1987.

[22] V.-D. Nguyen. Constructing stable grasps. Int. J. of Robotics Research, 8(1):26-37, 1989.

[23] G. A. Pfeifer and F. W. Liou. Investigation resulting in guidelines to optimize fluidized-bed fixture performance. In Proc. $2^{\text {nd }}$ ASME Conf. on Flexible Assembly Systems, pages 37-44, Chicago, IL, Sept. 1990.

[24] J. Ponce. On planning immobilizing fixtures for 3d polyhedral parts. In IEEE Int. Conf. on Robotics and Automation, pages 509-514, 1996.

[25] J. Ponce, J. W. Burdick, and E. Rimon. Computing the immobilizing three-finger grasps of planar objects. Proc. $2^{n d}$ Workshop on Comp. Kinematics, 1995.

[26] E. Rimon and J. W. Burdick. Mobility of bodies in contactii: How forces are generated by curvature effects. In Proc. Int. Conf. on Robotics and Automation, pages 2336-2341, San Diego, CA, May 1994.

[27] E. Rimon and J. W. Burdick. Mobility of bodies in contacti: A $2^{\text {nd }}$ order mobility index for multiple-finger grasps. IEEE Trans. Robotics and Automation, 14(5), 1998.

[28] E. Rimon and J. W. Burdick. Mobility of bodies in contactii: How forces are generated by curvature effects. IEEE Trans. Robotics and Automation, 14(5), 1998.

[29] J. C. Trappey, D. S. Su, and J. L. Hou. Computer-aided fixture analysis using finite element analysis and mathematical optimization modeling. In Proc. ASME Int. Mechanical Eng. Congress and Exposition, pages 777-787, New York, NY, 1995

[30] J. C. Trinkle. On the stability and instantaneous velocity of grasped frictionless objects. IEEE Trans. on Robotics and Automation, 8(5):560-572, 1992.

[31] A. Wallack and J. Canny. Planning for modular and hybrid fixtures. In Proc. Int. Conf. on Robotics and Automation, pages 520-527, San Diego, CA, May 1994.

[32] N. Xydas and I. Kao. Modeling of contact mechnics and friction-limit-surfaces for soft fingers in robotics, with experimental results. Int. J. of Robotics Research, 18(8):941-950.

[33] Y. Zhuang, K. Y. Goldberg, and Y.C. Wong. Planning for modular and hybrid fixtures. In Proc. Int. Conf. on Robotics and Automation, pages 543-549, San Diego, CA, May 1994. 